\title{
Economic and Disease Burden of Dengue in Mexico
}

\author{
Undurraga et al. 2015, PLoS Neglected Tropical Diseases
}

Here we present the STROBE checklist for the Morelos Cohort Study. We used some of the main results from this cohort study to estimate the economic and disease burden of dengue in Mexico. Not all categories in the STROBE checklist are applicable. As the primary purpose of this article is not to report the results of the Morelos Cohort Study, a separate manuscript including study design, specific objectives, results, and limitations of the Morelos Cohort Study is currently under preparation. A protocol of the study was published in 2012 [1], and is included in the references of the article.

S2 Table. STROBE checklist.

\begin{tabular}{|c|c|c|c|}
\hline & $\begin{array}{l}\text { Item } \\
\text { No }\end{array}$ & Recommendation & Status \\
\hline \multirow[t]{2}{*}{ Title and abstract } & \multirow[t]{2}{*}{1} & $\begin{array}{l}\text { (a) Indicate the study's design with a commonly used term in the title or } \\
\text { the abstract }\end{array}$ & $\checkmark$ \\
\hline & & $\begin{array}{l}\text { (b) Provide in the abstract an informative and balanced summary of what } \\
\text { was done and what was found }\end{array}$ & $\checkmark$ \\
\hline \multicolumn{4}{|l|}{ Introduction } \\
\hline Background/rationale & 2 & $\begin{array}{l}\text { Explain the scientific background and rationale for the investigation being } \\
\text { reported }\end{array}$ & $\checkmark$ \\
\hline Objectives & 3 & State specific objectives, including any prespecified hypotheses & N.A. \\
\hline \multicolumn{4}{|l|}{ Methods } \\
\hline Study design & 4 & Present key elements of study design early in the paper & $\checkmark$ \\
\hline Setting & 5 & $\begin{array}{l}\text { Describe the setting, locations, and relevant dates, including periods of } \\
\text { recruitment, exposure, follow-up, and data collection }\end{array}$ & $\checkmark$ \\
\hline \multirow[t]{2}{*}{ Participants } & \multirow[t]{2}{*}{6} & $\begin{array}{l}\text { (a) Give the eligibility criteria, and the sources and methods of selection of } \\
\text { participants. Describe methods of follow-up }\end{array}$ & $\checkmark$ \\
\hline & & $\begin{array}{l}\text { (b) For matched studies, give matching criteria and number of exposed and } \\
\text { unexposed }\end{array}$ & N.A. \\
\hline Variables & 7 & $\begin{array}{l}\text { Clearly define all outcomes, exposures, predictors, potential confounders, } \\
\text { and effect modifiers. Give diagnostic criteria, if applicable }\end{array}$ & $\checkmark$ \\
\hline $\begin{array}{l}\text { Data sources/ } \\
\text { measurement }\end{array}$ & $8 *$ & $\begin{array}{l}\text { For each variable of interest, give sources of data and details of methods } \\
\text { of assessment (measurement). Describe comparability of assessment } \\
\text { methods if there is more than one group }\end{array}$ & \\
\hline Bias & 9 & Describe any efforts to address potential sources of bias & $\checkmark$ \\
\hline Study size & 10 & Explain how the study size was arrived at & $\checkmark$ \\
\hline Quantitative variables & 11 & $\begin{array}{l}\text { Explain how quantitative variables were handled in the analyses. If } \\
\text { applicable, describe which groupings were chosen and why }\end{array}$ & $\checkmark$ \\
\hline \multirow[t]{5}{*}{ Statistical methods } & \multirow[t]{5}{*}{12} & $\begin{array}{l}\text { (a) Describe all statistical methods, including those used to control for } \\
\text { confounding }\end{array}$ & $\checkmark$ \\
\hline & & (b) Describe any methods used to examine subgroups and interactions & N.A. \\
\hline & & (c) Explain how missing data were addressed & N.A. \\
\hline & & (d) If applicable, explain how loss to follow-up was addressed & N.A. \\
\hline & & (e $)$ Describe any sensitivity analyses & $\checkmark$ \\
\hline
\end{tabular}

\section{Results}

Participants
$13 *$

(a) Report numbers of individuals at each stage of study - eg numbers 
potentially eligible, examined for eligibility, confirmed eligible, included

in the study, completing follow-up, and analysed

(b) Give reasons for non-participation at each stage

(c) Consider use of a flow diagram

\begin{tabular}{|c|c|c|c|}
\hline \multirow[t]{3}{*}{ Descriptive data } & \multirow[t]{3}{*}{$14^{*}$} & $\begin{array}{l}\text { (a) Give characteristics of study participants (eg demographic, clinical, } \\
\text { social) and information on exposures and potential confounders }\end{array}$ & $\checkmark$ \\
\hline & & $\begin{array}{l}\text { (b) Indicate number of participants with missing data for each variable of } \\
\text { interest }\end{array}$ & N.A. \\
\hline & & (c) Summarise follow-up time (eg, average and total amount) & $\checkmark$ \\
\hline Outcome data & $15^{*}$ & Report numbers of outcome events or summary measures over time & $\checkmark$ \\
\hline \multirow[t]{3}{*}{ Main results } & \multirow[t]{3}{*}{16} & $\begin{array}{l}\text { (a) Give unadjusted estimates and, if applicable, confounder-adjusted } \\
\text { estimates and their precision (eg, 95\% confidence interval). Make clear } \\
\text { which confounders were adjusted for and why they were included }\end{array}$ & $\checkmark$ \\
\hline & & $\begin{array}{l}\text { (b) Report category boundaries when continuous variables were } \\
\text { categorized }\end{array}$ & $\checkmark$ \\
\hline & & $\begin{array}{l}\text { (c) If relevant, consider translating estimates of relative risk into absolute } \\
\text { risk for a meaningful time period }\end{array}$ & N.A. \\
\hline Other analyses & 17 & $\begin{array}{l}\text { Report other analyses done-eg analyses of subgroups and interactions, } \\
\text { and sensitivity analyses }\end{array}$ & N.A. \\
\hline \multicolumn{4}{|l|}{ Discussion } \\
\hline Key results & 18 & Summarise key results with reference to study objectives & $\checkmark$ \\
\hline Limitations & 19 & $\begin{array}{l}\text { Discuss limitations of the study, taking into account sources of potential } \\
\text { bias or imprecision. Discuss both direction and magnitude of any potential } \\
\text { bias }\end{array}$ & $\checkmark$ \\
\hline Interpretation & 20 & $\begin{array}{l}\text { Give a cautious overall interpretation of results considering objectives, } \\
\text { limitations, multiplicity of analyses, results from similar studies, and other } \\
\text { relevant evidence }\end{array}$ & $\checkmark$ \\
\hline Generalisability & 21 & Discuss the generalisability (external validity) of the study results & $\checkmark$ \\
\hline \multicolumn{4}{|c|}{ Other information } \\
\hline Funding & 22 & $\begin{array}{l}\text { Give the source of funding and the role of the funders for the present study } \\
\text { and, if applicable, for the original study on which the present article is } \\
\text { based }\end{array}$ & $\checkmark$ \\
\hline
\end{tabular}

N.A. denotes not applicable.

Note: An Explanation and Elaboration article discusses each checklist item and gives methodological background and published examples of transparent reporting. The STROBE checklist is best used in conjunction with this article (freely available on the Web sites of PLoS Medicine at http://www.plosmedicine.org/, Annals of Internal Medicine at http://www.annals.org/, and Epidemiology at http://www.epidem.com/). Information on the STROBE Initiative is available at http://www.strobe-statement.org.

\section{References}

1. Martínez-Vega RA, Danis-Lozano R, Velasco-Hernández J, Díaz-Quijano FA, GonzálezFernández M, et al. (2012) A prospective cohort study to evaluate peridomestic infection as a determinant of dengue transmission: Protocol. BMC Public Health 12: 262. 